Rossini, V. \& Peiró-i-Gregòri, S. (2015). Educación en valores en la escuela italiana y española, desde la perspectiva de educar ciudadanos. Revista Electrónica Interuniversitaria de Formación del Profesorado, 18 (3), 113-125.

DOI: http://dx.doi.org/10.6018/reifop.18.3.190031

\title{
Educación en valores en la escuela italiana y española, desde la perspectiva de educar ciudadanos
}

\author{
Valeria Rossini ${ }^{(1)}$, Salvador Peiró-i-Gregòri ${ }^{(2)}$ \\ Universidad de $\operatorname{Bari}^{(1)}(\mathrm{IT})$, Universidad de Alicante (ES) ${ }^{(2)}$
}

\section{Resumen}

Nuestro análisis se centra en la importancia de los valores en la educación en el contexto de un mundo globalizado. Según lo recomendado por las directrices de la UE sobre el desarrollo de las competencias personales, interpersonales, interculturales, este tipo de educación se finalice para apoyar el respeto de los derechos humanos y el concepto de "ciudadanía activa". Entre todos los países europeos, consideramos el caso de Italia y España, observando los esfuerzos de sus sistemas educativos en la conjunción de las virtudes humanas (paideia) con las virtudes sociales (polity) dentro de la escuela, a través de valores como la justicia y la solidaridad, la libertad, la igualdad, el pluralismo y la participación. Todos estos valores, defendidos por sus maestros, deben ser la base para una buena convivencia entre los estudiantes, como modelo para su vida futura de ciudadanos activos. Para este propósito los alumnos tienen que estar más involucrados en situaciones prácticas, aprendendo cómo "permanecer en el mundo".

\section{Palabras clave}

Valores, educación, escuela, ciudadanía activa.

\section{Values education in the Italian and Spanish school, from the perspective of educating citizens}

\section{Contacto:}

Valeria Rossini, valeria.rossini@uniba.it, Departamento de Ciencias de la Educaciòn, Psicologìa, Comunicaciòn, Universidad de Bari. Palazzo Ateneo, Piazza Umberto I, 1 - 70100 BARI (IT). El artículo ha sido compartido por los autores. Valeria Rossini escribió los apartados 1 y 2, Salvador Peiró-iGregòri los apartados 3 y 4. 


\section{Abstract}

Our analysis is focused on the importance of values in education in the context of a globalized world. As recommended by EU guidelines about the development of personal, interpersonal, intercultural competencies, this kind of education is finalized to support the respect for human rights and the concept of "active citizenship". We consider, among all European countries, the case of Italy and Spain, observing their educational systems'efforts in conjuncting human virtues (paideia) with social virtues (politeia) inside school, through values as justice and solidarity, freedom, equality, pluralism and participation. All these values, upholded by their teachers, should be the basis for a good coexistence among students, as a model for their future life of active citizens. For this purpose, pupils need to be more involved in practical situations, learning how "to stay in the world".

\section{Key words}

Values, education, school, active citizenship.

\section{Introducción}

Desde la perspectiva histórica, los valores constituyen el humus vital de la reflexión pedagógica, especialmente a partir de finales del siglo XIX. En esto, hay que tener en cuenta la llamada «vuelta a Kant», en virtud de lo cual, algunas alas de la cultura filosófica alemana se comprometieron a reivindicar los derechos de la libertad humana contra el predominio de leyes analíticas y clasificadoras de matriz positivista, siguiendo las huellas de Windelband, Rickert, Scheler, Hartmann. Todo lo cual influyó en las reformas educacionales decimonónicas, sin ahorrar un debate entre los modelos ilustros filantropistas y los neohumanistas (Martí, 2012). Estos últimos plantean compensar la suficencia del racionalismo, así tenemos la laborg de Herder y otros, que integraron las aportaciones enciclopédicas con los valores y cultura, suplementando así la anaxología ilustrada, como nos explica (Bergman, 1984).

La Unión Europea y la Recomendación del Consejo de 18.12.2006 sobre las competencias clave para el aprendizaje permanente cambia el enfoque en las habilidades sociales, como competencia personal, interpersonal e intercultural, que cubre todas las formas de comportamiento que preparan a las personas para que participen manera eficaz y constructiva en la vida social y laboral, sobre todo la vida en sociedades cada vez más diversas, así como para resolver los conflictos, cuando ello sea necesario. Por consiguiente, tenemos algunas inquietudes intelectuales en cunto a la preparación de futuros ciudadanos: ¿cómo lo enfocan los sistema educativos español e italiano?, ¿basta con complementar los contenidos enciclopédicos mediante "raciones" de valores?, ¿es que la persona se autorrealiza sólo con nociones más valores, asimilados a modo de yuxtaposición de éstos?, etc.

\section{Los valores entre educación e instrucción}

La educación, siempre asociada a los valores, ha venido configurándose históricamente como el proceso de construcción de una forma interior a través de la maduración personal, mediante la adquisicíón comprensiva de lo cultural, debidas al crecimiento ético y cognitivo del sujeto. 
Con el tiempo se ha ido aflojando el estrecho vínculo entre dimensión ética y dimensión cognitiva ínsito en la idea de paideia, abriéndole paso a una separación entre la adquisición del saber, que se realiza gracias a la instrucción, a la formación moral, y al fortalecimiento del carácter generado por la educación (Peiró, 2013).

De toda manera, la educación no puede prescindir de los valores (Peiró, 2000) y se le exige que promueva situaciones para que el educando los encarne. Es decir, entablar una relación en la que el educador tiene la tarea de proponer un marco valoral, mientras que al educando le corresponde luego decidir hacerlo propio, para así ir construyendo sy personalidad.

Constatamos, así, la inseparabilidad de educación y valores, los cuales actúan como un aspecto vitalizador de cada acción educante. Pero, queda por especificar y compartir un posible significado pedagógico para el término valor: es ésta una operación nada fácil, considerando su peculiar ambigüedad polisémica.

El término valor adquiere acepciones diferentes en los distintos enfoques disciplinares: en economía indica el precio de un objeto, se relaciona con el concepto de utilidad y costes de producción y conlleva la distinción entre valor en uso y valor de cambio (GEE; Samuelson \& Nordhaus, 2008). En sociología (Rueff, 1964) denota un objeto de deseo condicionado y distribuido culturalmente según niveles de preferencia. En este sector puede leerse el valor tanto desde una perspectiva predominantemente emocional como desde una óptica esencialmente moral. Por ejemplo, según opinan Thomas y Znaniecki (1996), el valor es una entidad dotada de significado o bien algo que ejerce una fuerte atracción emocional sobre el individuo -en este último caso coincidiría con necesidad, deseo o interés. Para Weber (2004) el valor es el principio orientador de la conducta, con fuerte connotación moral. Sin embargo, el sociólogo alemán opina que el hombre -en cuanto ser social libre- define los valores de manera puramente subjetiva y autónoma. Esta libertad decisoria desemboca en un «politeísmo de valores» que acaba por convertirse inevitablemente en antagonismo y conflictividad entre valores contrapuestos. En filosofía el lema expresa la cualidad por la que una cosa posee dignidad y, por consiguiente, es digna de estimación y respeto (Marín, 1976). De ahí que se perciba el valor como aquello por lo cual un ser es digno de ser, una acción es merecedora de realización. También en este sector no faltan posiciones críticas del concepto tradicional de valor como instrumento de adecuación conformista a la ideología burguesa y a la moral dominante (nos referimos a la escuela de Francfurt, o al propio Heidegger). En psicología se considera el valor como motor de acción: en opinión de Maslow (1943), lo valoral está vinculado a la respuesta gratificadora a las necesidades fundamentales genéticamente diferentes en la edad evolutiva. Más generalmente, en términos psicológicos el valor indica cualidades de la personalidad, a saber actitudes, disposiciones, convicciones, creencias relativas a los bienes que los hombres juzgan positivos y al orden jerárquico subjetivo de dichos bienes (Maslow, 1943).

Es posible asociar al concepto de valor todo aquello a lo cual el individuo está dispuesto a conceder una importancia tan alta como para que llegue a ser fuerza organizadora de su comportamiento. Desde este punto de vista, el valor se revela no sólo como principio de evaluación, sino también como fuente de compromiso y motor de acción.

La idea de autonomía funcional (valores) llevó a Allport (1965) y a sus seguidores Vernon \& Lindzey (1972) a desarrollar una categoría de valores. De acuerdo con Allport, la necesidad de valor corresponde a la necesidad del hombre de hacer referencia, a la hora de tomar decisiones, a entidades según las cuales ordenar y justificar sus elecciones, intentando, gracias a éstas, encontrarle "sentido» a su propia vida y asumiendo que son los valores elegidos lo que la hacen sensata. «En plural, indica ideales, ideas-fuerza, que se proponen 
como humanamente dignos de por sí y humanizantes en su realización» (Prellezo, Nanni \& Malizia, 1997, 1155).

Los valores se convirtieron en objeto de reflexión pedagógica en relación con los conceptos de «deber ser», "verdad», «orientación normativa». "De hecho, actuar en función de los valores significa establecer la comparación con un «deber ser», o bien un «no deber ser», esto es, atribuir un significado en relación con una experiencia personal y/o colectiva» (Chiosso, 2004, 89).

Además, al considerar el valor como todo «lo que vale», se corre el riesgo de limitarse a una definición tautológica que nada dice acerca de las razones por las que «lo que vale» se considera tal. «Lo que sí vale para mí » puede no valer para el otro; por tanto, es necesario definir qué es «lo que para nosotros vale» basándonos en una razonable negociación de sentido. Para conjurar el riesgo de un subjetivismo que deja al individuo solo frente a la elección y adhesión a los valores que pueden dar sustancia a su vida, una posible teoría pedagógica de los valores podría fundamentarse en la etimología griega del término. En el vocabulario griego, el valor se denomina axia, procedente del verbo ago, e indica «lo que con su peso tira o contrabalanza» (Cattanei, 1991, 23). Visto así, aparece el valor como algo que atrae en virtud de su fuerza: diferentemente del deseo, que se refiere al ámbito de lo inmediato y momentáneo, el valor se relaciona con las dimensiones de solidez y perennidad. Según Cacciaguerra $(1990,25)$ «el valor es el concepto estable de lo que es deseable», entendiendo por concepto una convicción duradera y no extemporánea, una actitud que involucra razón, sentimiento y emotividad a la vez.

Tradicionalmente, se considera la escuela como la agencia formalmente encargada de la transmisión del saber. Por lo tanto, su función más peculiar coincide con la instrucción, suministrada según criterios laicos y no ideológicos. Entonces, si los valores no pueden convertirse en objetos de enseñanza por el simple hecho de referirse precisamente al plano educativo; la instrucción, por ser algo diferente de la educación, no debería incluir en sus contenidos y modalidades la dimensión axiológica. Esta consideración deja sin respuesta al menos dos preguntas. La primera se plantea la cuestión de si es posible considerar el ámbito didáctico y el pedagógico como realidades separables y/o autónomas. La segunda, directa consecuencia de la anterior, tiene que ver con la supuesta neutralidad del conocimiento. Ambas se remiten a las distintas ideas de educación e instrucción, así como sus diferencias.

En la acepción más común, el término instrucción indica la adquisición de conocimientos, informaciones, nociones. Se refiere esencialmente al sector del saber. En cambio, muy a menudo se emplea el término educación para indicar la adquisición de actitudes y capacidades que atañen tanto al comportamiento moral (educación moral) como a otras dimensiones de la personalidad (educación social, afectiva, lingüística, etc.). Por lo que, en esta última acepción, se trata en mostar maneras de vivir esas nociones-fuerza.

Por lo tanto, la instrucción (que procede de la palabra latina instruere, es decir, habilitar, preparar a alguien para algo) hace referencia a la esfera de lo cognitivo y a los procesos de adquisición de conocimientos, saberes y competencias (analíticas y descriptivas, interpretativas y críticas, heurísticas y creativas). La educación (en su acepción doble que procede de las dos palabras latinas: educāre, que significa criar, cebar; e-ducĕre, que significa extraer o sacar de) se refiere sobre todo al ámbito de reflexión valorial, ético-social y se expresa y realiza especialmente en las instituciones no formales, a saber familia, iglesia, libres asociaciones y, claro está, la escuela (Frabboni y Pinto Minerva, 2003).

La escuela, por ser lugar no tan sólo de instrucción, sino también de educación de los menores, la escuela no puede quedarse indiferente ante el dato axiológico. Según estas 
ideas, la institución docente cumple con sus deberes educativos a través de la enculturación y socialización de las nuevas generaciones. A pesar de que se caracteriza por el respeto a la autoridad formal y por dinámicas con alto grado de oficialidad y neutralidad afectiva, el ambiente escolar es (o debería ser siempre) modelo de democracia, laboratorio de paz, taller de valores (Rossini, 2011).

No obstante, entre la instrucción sin valores y el desempeño de tales, por aprendizaje, hay un vacío, al menos conceptual. Por esta razón, hay que hablar de un algo situado entre lo noético y lo volitivo, que responda a esta inquietud pedagógica: es la formación. Los valores en los que uno se forme darán sentido a lo asimilado por instucción.

En efecto, los procesos de enseñanza-aprendizaje no se pueden separar de las experiencias afectivas y relacionales con las que los estudiantes, en la vida escolar de cada día, aprenden a cuidar de sí mismos y de los demás, ocuparse del medio ambiente y resolver problemas y conflictos interpersonales. Por esto, las prácticas didácticas deberían proponerse como aproximación $a$, reflexión sobre, testimonio de conjuntos axiológicos que hay que proteger y defender, evitando por supuesto que dicha actividad de protección y defensa degenere en actitudes de cerrazón e intolerancia hacia otros posibles universos de valores.

Por lo tanto, los valores se convierten en válidos puntos de referencia para desarrollar el sentido de legalidad, practicar la convivencia civil y participar en la vida comunitaria. Sin embargo, son muchos los que creen que la intervención intencional y sistemática de la escuela en el ámbito de las opciones éticas de los alumnos puede limitar, de alguna manera, la libertad individual y producir el riesgo de catequización, adoctrinamiento e ideologismo. De hecho, una escuela que educa en valores podría ser tachada de ser un lugar en el que se adoctrina a las nuevas mentes, se les inculca ideas absolutas e inmodificables con vistas a manipularlas y someterlas

La verdad es que la educación en valores no coincide con la educación moral (y menos aún con la religiosa); por otra parte, también en el caso de que fuera vehiculada por contenidos morales y religiosos, la educación no podría degenerar jamás en adoctrinamiento, con tal de que se imparta respetando las opiniones y elecciones de la persona.

Sólo bastaría con recordar el significado de la palabra indottrinamento (adoctrinamiento) para comprender lo lejos que está de los objetivos de la escuela, si bien, al nivel etimológico, se coloque muy cerca de enseñar. Según el diccionario Hoepli, indottrinare (adoctrinar) significa enseñar los principios fundamentales de una doctrina, una ideología, por lo general política, especialmente de manera dogmática. Muy similar la definición ofrecida por el diccionario Treccani, según el cual el verbo equivale a instruir, hacer asimilar, gracias a prácticas de enseñanza y persuasión persistentes y metódicas, los principios de una doctrina, sobre todo política. Así, el término se emplea principalmente con tono irónico y polémico. Además, el Dizionario della Lingua Italiana Le Monnier añade que indottrinare es someter a un incesante martilleo ideológico. En las investigaciones que examinan los movimientos de propaganda política y las sectas religiosas, incluso se asocia el adoctrinamiento al lavado de cerebro o a la reforma y control del pensamiento (Lifton, 1989).

Educando para ejercer el pensamiento crítico y a la autonomía intelectual, la escuela es incompatible tanto con el adoctrinamiento de este tipo como con otras formas más generales de persuasión (Esteve, 1981).

Si entendemos, por ejemplo, la enseñanza como el acto de imprimir signos, ésta podría parecerse al acto de inculcar, que de hecho significa imprimir profunda y persistentemente algo en el alma y la mente de alguien. La etimología latina (in-calcare) remite materialmente 
al acto de introducir forzosamente y, en sentido traslaticio, corresponde a situar un consejo o una advertencia en la memoria, grabar en la mente.

En la sociedad de la globalización y de la información, la educación en valores constituye un instrumento fundamental de ejercicio y defensa de los derechos humanos y, al mismo tiempo, guía el desarrollo individual y social. Educación y formación para ejercer la ciudadanía representan un binomio esencial en los currículos escolares ya en los primeros niveles de instrucción, así como uno de los instrumentos fundamentales para realizar esa idea nueva de educación para los años dos mil (Delors, 1997).

Los estudios internacionales sobre educación cívica y para la ciudadanía ponen de relieve la necesidad de averiguar no sólo los conocimientos de los alumnos acerca de estos temas, sino también, y sobre todo, sus actitudes y comportamientos en ámbito escolar y extraescolar. "Como la Educación para la Ciudadanía es una materia orientada al desarrollo de la reflexión crítica del alumnado para que construyan un pensamiento y un proyecto de vida propio, creemos que sería conveniente, además de necesario, la presentación de alternativas de acción e implicación que permitan a aquéllos valorar y decidir acerca de su compromiso vital, con información suficiente y ofreciendo de múltiples puntos de vista. A título de ejemplo, el Proyecto ICCS 2009 (International Civic and Citizenship Education Study), iniciado en 40 países del mundo, se propone «identificar e indagar, dentro de un marco comparativo, cómo se prepara a los jóvenes para que desempeñen de manera activa su papel de ciudadanos en sociedades democráticas».

La escuela cumple este cometido por medio de recorridos de conocimientos, reflexión y acción, que repercuten inmediatamente en la calidad del estar juntos en clase: la coherencia entre lo que se estudia y lo que se vive en la cotidianidad de las experiencias educativodidácticas es el indicador más relevante de la eficacia formativa en tal dirección.

En Italia, la educación en valores en ambiente escolar se ha traducido en una especie de currículo transversal o suplementario capaz de influir sobre todo en las dimensiones cognitiva y social del alumnado. Esta ha sido fundamentalmente asociada a la educación cívica, que la Ley 169/2008 ha transformado en la asignatura "Cittadinanza e Costituzione" (Ciudadanía y Constitución), perteneciente a las áreas histórico-geográfica e histórico-social. En las intenciones del legislador, educar para la ciudadanía equivale a promover la capacidad para sentirse ciudadanos activos, que ejercen sus derechos inviolables y cumplen los deberes inderogables en la sociedad de la que son miembros. Además, el estudio de la Constitución permite conocer el documento fundamental de nuestro ordenamiento democrático y sacar de él una serie de valores útiles para ejercer los derechos/deberes de ciudadanía. Por lo que se suplental los procedimientos instructivos para promover procesos formativos en los escolares.

La Constitución italiana hace explícita referencia a algunos valores importantes que son elementos imprescindibles en la convivencia escolar: la solidaridad y la justicia. En el Artículo 2 se afirma que «la República reconoce y garantiza los derechos inviolables del hombre, ora como individuo, ora en el seno de las formaciones sociales donde aquél desarrolla su personalidad, y exige el cumplimiento de los deberes inexcusables de solidaridad política, económica y social». Además, el Artículo 3 añade que «todos los ciudadanos tendrán la misma dignidad social y serán iguales ante la ley, sin distinción de sexo, raza, lengua, religión, opiniones políticas ni circunstancias personales y sociales». Como la escuela es un sistema, ya que es una institución del estado, es necesario que en sus aulas se promuevan estos valores, a través de recorridos educativos que logren repercutir en las dimensiones más profundas de niños y adolescentes, así como en el plano de la afectividad, la moralidad y la espiritualidad. 
Con relación a España, partimos explicando la ética mínima que la Constitución de 1978 y la vigente Ley - LOE - de educación definen. Considerando los diversos apartados que sobre lo axiológico refiere la carta magna (Constitución Española), tenemos las siguientes proposiciones: libertad, justicia, igualdad; pluralismo político; participación de todos los ciudadanos en la vida política, económica, cultural y social.

No obstante, la nueva reforma de la escuela, puesta en marcha por la Ley Orgánica para la Mejora de la Calidad Educativa - LOMCE -, parece que sustituye lo anterior por la fijación curricular de la asignatura de Religión, dándole plena validez académica, aunque también se establece una materia "alternativa fuerte" a la misma, que se llamará Valores Culturales y Sociales en primaria y Valores Éticos en secundaria. Por otro lado desaparece definitivamente la materiaEducación para la ciudadanía, donde se materializa la competencia social y ciudadana (Ortega Gaite, Tejedor Mardomingo, Ruiz Ruiz, 2012). Esto significaría que, en vez de plantear valores "sueltos", tales se contextualizan dentro de una sistematización lógica curricular. También se formula un porcentaje de competencias básicas que, tal vez, incluya ciertos valores.

En los textos mencionados legislativos se ha reiterado la apelación a la persona del educando. Pero, si en tales párrafos se explicitan los valores, digamos, socio-políticos, no queda claro la referencia a los humanísticos. Sin embargo, pueden integrarse ambos. Un ejemplo puede ser el siguiente (figura $\mathrm{n}^{\circ} 1$, desde abajo hacia arriba): para desenvolverse en una convivencia en paz se requiere antes que se viva la democracia en libertad. Sin embargo, esto es extremadamente difícil sin un clima de justicia. Pero, para que uno sea justo, ha de ser generoso, pero esto requioere antes comprender; no obstante, uno no comprenderá aotro si no reconoce sus limitaciones (humildad). Este esquema sólo pretende ofrecer una intuición que muestre la necesidad de fundamentar las virtudes humanas (paideia) con las sociales (politeia), siendo las humanísticas condición para que las políticas no sean huecas, mera palabra.

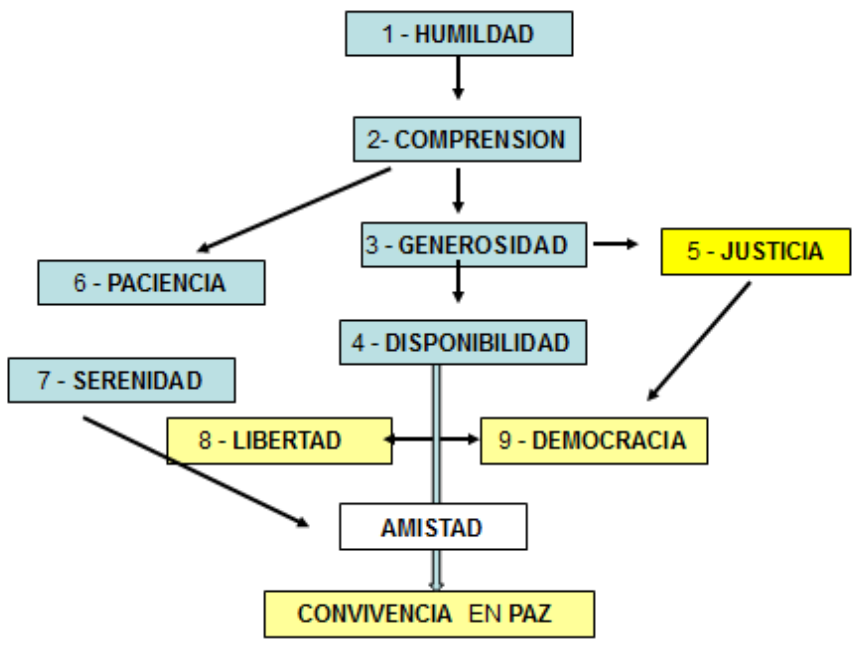

Figura 1. Convivir como resultado de integrar las fortalezas humanas para promover la convivencia ciudadana.

\section{Lineamientos de un modelo pedagógico}

Tras el gran rechazo realizado por las pedagogías antiautoritarias y libertarias, el tema de la relación entre escuela y valores ha vuelto a imponerse en la escena pedagógica casi como 
una emergencia. Si por un lado el incremento del nivel general de instrucción hace a la población juvenil más sensible a la complejidad social, más abierta, reflexiva y crítica hacia las instituciones políticas y, por tanto, propensa a ocuparse de la vida pública y a participar activamente en el funcionamiento de la democracia; por otro lado, acreditados análisis psicosociales de la condición juvenil nos hablan de una generación en profunda crisis de identidad (Agulló, 1997; Penas, 2008). Así, con ser más inteligentes $-\mathrm{Cl}$ - que sus coetáneos del pasado, los jóvenes de hoy parecen ser sujetos caracterizados por un individualismo narcisista y un resignado desinterés. Indiferentes hacia el bien común, ajenos a las dinámicas políticas, aplastados en el presente, son cada vez más frágiles y desorientados ante los procesos decisionales que los afectan.

Como dice Penas (2008) desde la institución escolar, apreciamos que la educación en valores es entonces imprescindibile para preparar también a los casi adultos alumnos de secundaria (que están para alcanzar la mayor edad) para que entren como protagonistas en la escena político-social con una proyectualidad individual y social bien definida. Por consiguiente, los adultos - particularmente padres y docentes - tienen que ser fieles a su tarea educativa, aunque poco a poco su papel se haya debilitado, debido a la deslegitimación social, una difusa permisividad y una condición existencial objetivamente difícil.

En la escuela, la relación entre docentes y alumnos quizá se halle aún más llena de implicaciones pedagógicas, por estar menos mediada por la dimensión de relativa a interacciones primaris, como es el caso de la familia, por lo que amortigua mejor los ineludibles conflictos intergeneracionales. En los contextos escolares, a los docentes les cuesta presentarse como puntos de referencia y maestros de vida, y esta incomodidad también perjudica la bondad de su enseñanza, pues produce repercusiones negativas en el nivel de motivación de los alumnos y en el clima sociorelacional del grupo-aula. En esta lógica, educar en valores en la escuela significa enseñar a los alumnos, en tanto que próximos ciudadanos, a cómo estar en el mundo (en la polis).

Por lo explicado más arriba, este objetivo no se puede alcanzar recurriendo sólo a formas transmisivas, por ejemplo la clase frontal tradicional. Desde esta perspectiva, los valores representan, más que temas, ámbitos experienciales, por esto se conciben como transversales (Marín, 1975; Peiró, 1979), que trascienden las distintas áreas disciplinares y afectan a la dimensión explícita e implícita, patente y oculta, emergida y subterránea de la didáctica.

Un primer posible recorrido educativo basado en valores hace referencia a las pautas de educación moral que hay que insertar dentro de un currículo específico, como ya propusieron Maritain (1935 y 1981) y otros autorizados estudiosos a mediados del siglo XX. Por consiguiente, optar por este tipo de recorrido presupone la elaboración de una «didáctica de la moral» en la cual sea posible disponer de una progresión en la elección de contenidos y metodologías de enseñanza, que discurran no sólo consecuentemente con el nivel de maduración del alumno y el estado de sus conocimientos, sino con la facilicitación de los actos de libre juicio y toma de decisiones autónomas.

Sin querer, de ninguna manera, desconocer la importancia de reflexionar sobre los valores partiendo de las disciplinas de estudio, también se podría pensar en un trabajo más indirecto, pero quizás más profundo, que actúe al nivel social e individual. Se trata de reflexionar sobre todo sobre aquellos valores en los que se fundamentan los pilares del proceso educativo escolar y a los que ya se ha remitido cuando hablamos del dictamen constitucional. 
a) El valor de la justicia en el manejo de la clase. Los docentes desempeñan un papel fundamental para la construcción del know-why, es decir el núcleo central de las competencias axiológicas. La relación con los alumnos no puede sino inspirarse en el respeto al otro, con una actitud no juzgante y que anima, en el diálogo empático, a través de una comunicación positiva y transparente, en la responsabilidad individual, a través del empeño constante en el trabajo. La relación educativa -en su sentido de relación de ayudaexplota positivamente la intencionalidad y la sistematicidad de la intervención educativa y sirve de muro de contención de eventuales comportamientos egocéntricos, agresivos y antisociales. A tal efecto, adquieren considerable importancia las prácticas referidas al respeto de las reglas y a los comportamientos causados por su transgresión. Aunque no se pueda reducir la educación en valores a manejo de reglas de conducta, los juicios de aprobación de las acciones tienen que ver con la imparcialidad, la coherencia y, en fin, con la aplicación de criterios de justicia en decisiones que atañen a la disciplina escolar. Con relación al tema de la justicia, los estudiantes le prestan mucha atención: defender este valor en la escuela quiere decir comprometerse a garantizar un trato ecuánime para todos los estudiantes, lo cual va a ser instrumento de cohesión en la clase y de fe en la autoridad de los docentes.

b) El valor de la solidaridad en las dinámicas sociorelacionales. La escuela educa en valores por medio de recursos pedagógicos y sociales, a saber el clima de clase, las relaciones de cooperación entre pares, la organización del entorno escolar. Estas condiciones integradas en el llamado curriculum oculto - hidden curriculum- , contribuyen a transmitir mensajes implícitos cargados de contenido valorial. Particularmente, las normas que el grupo-aula se esfuerza por negociar y respetar dependen de la estructura sociorelacional a menudo invisible que une a los alumnos en una relación de íntima interdependencia. La importancia de este conjunto de reglas informales radica en el hecho de que ellas influyen en la atmósfera que se respira en el aula, orientando las interacciones entre alumnos en sentido cooperativo o competitivo. Las normas del grupo-aula son reglas que suelen estar tan presentes como los reglamentos escolares, puesto que éstas canaliza los comportamientos de las situaciones concretas. A raíz de ellas, se plantean juicios de aceptación o exclusión (considerar la gama intermedia) hacia los alumnos» (Peiró, 2005, 140).

El conjunto de motivaciones, actitudes, convicciones implícitas y explícitas sobre el estar juntos en la escuela parece repercutir notablemente en la disposición de los alumnos para crecer juntos, desarrollando el sentido de pertenencia a su grupo-aula que debería prevenir episodios de crispada conflictividad que ocasionan problemas de convivencia escolar.

En cuanto a los profesores y al ejercicio de su autoridad, la educación no puede tapar el conflicto mediante actuaciones docentes emocionalizadas (Wojtyla, 2011). Los valores humanísticos contribuyen a promover el carácter de cada escolar. Hay que ver cómo se lleva a cabo tal contenido en escuelas con éxito. Bennett et alii (1999) sintetiza la educación de la personalidad mediante una frase clave: La buena enseñanza del carácter cultiva virtudes mediante la formación de buenos hábitos. Según Bennett, los niños deben aprender mediante actos que la honestidad y la compasión son buenas, y que el engaño y la crueldad son malos. El autor propone acciones educativas más vitales en donde los adultos deben esforzarse por ser modelos de buen carácter. La enseñanza de los valores es más efectiva si se integra en los diversos aspectos curriculares. Por ejemplo, en Ciencias, los maestros pueden discutir el valor de la honestidad en los datos. En Matemáticas los estudiantes pueden aprender la perseverancia trabajando en un problema hasta dar con la solución correcta. La Historia también contiene lecciones valiosas, como pueden ser el estudio de personajes por épocas, interpretando su carácter. 
Pero, aparte de lo que decimos en el libro reciente (Peiró, 2013), hay otros modelos efectivos. No todos los programas de enseñanza de valores son efectivos, según Bennett (1999). Las discusiones sobre control de armas, aborto y matrimonio gay no les enseñan a los jóvenes sobre el bien y el mal, ni a cómo llevarse bien con los otros. Los programas efectivos involucran a los niños con actividades prácticas en donde se enfatizan los valores en la escuela y en el currículo.

Pero, educar los hábitos para logar un carácter libre no excluye la excelencia académica. El modelo italiano de unir la enseñanza de ciertos valores con asignaturas se confirma en estas experiencias, a la vez que abren la posibilidad de que cada asignatura se suplemente con los valores humanísticos. La enseñanza de los valores también incluye tener altos estándares de éxito en exámenes. Cuando se hace trabajar y sudar mentalmente a los estudiantes, éstos aprenden virtudes como la laboriosidad y la perseverancia, constancia, dice Bennett. Por el contrario, asevera el autor, las escuelas estimulan la pereza, la despreocupación y la irresponsabilidad cuando los estudiantes rara vez tienen tareas, cuando no se les hace responsables por sus errores de ortografía o gramática o aritmética, cuando pueden obtener altas calificaciones con poco esfuerzo. Educar en valores significa conseguentemiente construir un ambiente resiliente a nivel individual y comunitario, «donde tanto los problemas de minor entitad come los urgentes se enfrentan mediante recursos colectivos y compartidos, puestos en circulación entre las personas en sinergia». (Marzana, Marta \& Mercuri, 2013).

Reintegrando aquí lo dicho antes sobre los valores y escuela-comunidad, hemos de repensar el rol de los padres y madres. Muchos maestros se quejan de que los padres o madres son muy permisivos y no proveen suficiente disciplina en casa. La enseñanza de los valores funciona mejor cuando las escuelas y las familias trabajan juntas.

\section{Discusión, conclusiones y propuestas.}

La legislación establece contenidos axiológicos para orientar la tarea educativa. Por tanto, reconoce y declara lo que por naturaleza efectúa y ha hecho la humanidad para educar a cada nueva generación. Pero, no es suficiente con legislar. Sólo con formular una serie de disposiciones jurídicas no significa que lo puesto "in jure" vaya a ser "in facto". «En los centros escolares continúan primando el currícolo y los conocimientos, a la trasmisión de valores» (Penalva, Hernández \& Guerrero, 2013). Tampoco la institución intermedia, que es rel proyecto educativo institucional o de centro - PEC - garantiza ese paso de lo establecido por el derecho positivo se encarne en los estudiantes. La preparación docente, junto con la suplementación con actividades extra/para-escolares es importante y conditio sine qua non.

Con las anteriores proposiciones, puedemos afirmar que enseñar valores en abstracto, entendidos como temas y/o desligados de la propia vida, podrían sólo formar los alumnos, pero no grantiza fortalecer el carácter, propiciar un voluntad más libre. No obstante, no hay que desperdiciar la enseñanza de los valores fundamentales, pues ;claro que dan sentido al conocer!, pero es insuficiente. Si una personalidad no está suficientemente madura, ajustad, pude ser esclava de su carácter. Por tanto, los valores humanos han de interrelacionarse con la vivencia de los sociales. Si esto no se hace, tales ideales pueden quedar en lo virtual. Primero, pues, hay que formar tal aspecto de la persona (Brody, 1995); luego y a la vez, contagiar e instruir en la tolerancia, la paz, la libertad, etc.

En resumidas cuentas, en una óptica preventivo-promocional la educación en valores se concreta también en la promoción de comportamientos prosociales en alumnos y docentes. Estos encarnan el valor de la solidaridad y acomodan el de justicia: esto impide 
que los aparatos de poder arraigados en la clase conduzcan al ajuste ortodoxo de sus dinámicas interiores hasta engendrar actitudes de excesivo conformismo u obediencia pasiva en el interior del grupo.

Desde este punto de vista, hay que tener cuidado para no confudir las acciones colaborativas, la ayuda mutua y la comprensión empática con que se manifiestan los valores de justicia y solidaridad, con las actitudes de complacencia, emulación o, aún peor, ley del silencio, que ofrecen terreno fértil para la cultivación de relaciones basadas en dinámicas de dominio/dependencia tanto entre docente y alumno como entre alumnos.

Una desmesurada complicidad entre docente y alumno y una excesiva cohesión en el grupo-aula no son indicadores de convivencia positiva y pacífica. Muy al contrario, a menudo ocultan el miedo al enfrentamiento y al conflicto, la tendencia a una inmovilidad que atrofia el desarrollo individual y social, al tiempo que fortalece las eventuales tendencias de algunos a la sumisión y al sometimiento.

\section{Bibliografía}

Agulló, E. T. (1997). Jovenes, trabajo e identidad. Oviedo: Universidad de Oviedo.

Allport, G.W. (1965). Pattern and growth in personality. New York: Holt, Rinehart \& Winston.

Allport, G.W., Vernon, P.E. \& Lindzey, G.E. (1972). Estudio de valores. México: El Manual Moderno.

Bennett, N., Dunne, E., \& Carré, C. (1999). Patterns of core and generic skill provision in higher education. Higher Education, 37, 71-93.

Bergman, A. (1984). L'épreuve de l'étranger. Culture et tradition dans l'Allemagne romantique: Herder, Goethe, Schlegel, Novalis, Humboldt, Schleiermacher, Hölderlin. París: Gallimard.

Brody, J. M. (1995). Carácter first. The American School Board Journal. San Francisco: JosseyBass Publishers.

Cacciaguerra, F. (1990). Il secondo sangue: la circolazione dei valori in famiglia. Enna: Oasi

Cattanei, G. (1991). Cultura dei bisogni, cultura dei valori. in L. Santelli Beccegato (ed.), Bisogno di valori. Per un rinnovato impegno educativo nella società contemporanea. Brescia: La Scuola.

Constitución Española (1978). Enlace web: http://www.uned.es [12-06-2013].

Chiosso, G. (2004). Teorie dell'educazione e della formazione. Brescia: La Scuola

Delors, J. (1997). Nell'educazione un Tesoro. Rapporto all'UNESCO della Commissione internazionale per il Ventunesimo secolo (traducción italiana). Armando: Roma

EACEA/Eurydice (2012a). Entrepreneurship Education at School in Europe: National Strategies, Curricula and Learning Outcomes. Bruxelles: Eurydice.

EACEA/Eurydice (2012b). Key data on education in Europe 2012. Bruxelles: Eurydice.

Esteve, J. M. (1981). Fuentes de tensiòn individuales y sociales con incidencia en la actuaciòn profesional del educador. En La calidad de la educaciòn. Madrid: Consejo Superior de Investigaciones Cientificas, 187-193. 
Frabboni F. \& Pinto Minerva F. (2003). Introduzione alla pedagogia generale. Roma-Bari: Laterza.

Galino, M. A. (1960). Historia de la Educación I. Edades Antigua y Media. Madrid: Gredos.

Indicazioni Nazionali per il curricolo della scuola dell'infanzia e del primo ciclo d'istruzione, de 4 de septiembre de 2012. Enlace web: http://www.istruzione.it. [12-06-2013].

Ley 169/2008. Enlace web: http://eur-lex.europa.eu. [12-06-2013].

Lifton, R. J. (1989). Thought Reform and the Psychology of Totalism. Nueva York: Norton Library.

LOE: Ley Orgánica 2/ 2006, de 3 de mayo, de Educaciòn. Enlace web: http://www.boe.es. [1206-2013].

Ley Orgánica para la Mejora de la Calidad Educativa - LOMCE.MK. Enlace web: http://www.mecd.gob.es [12-06-2013].

Marín Ibáñez, R. (1975). Interdisciplinaridad y enseñanza en equipo. Valencia: ICE-UPV.

Marín Ibáñez, R. (1976). Valores, objetivos y actitudes en educación. Valladolid: Miñón.

Maritain, J. (1935). Humanisme intégral. Problèmes temporels et spirituels d'une nouvelle chrétienté. Paris: Fernand Aubier.

Maritain, J. (1981). La educación en este momento crucial, Buenos Aires: Club de Lectores.

Martí Marco, M. R. (2012). Wilhelm von Humboldt y la creación del sistema universitrio moderno. Madrid: Verbum.

Marzana, D., Marta, E., Mercuri, F. (2013). De la resiliencia individual a la resiliencia comunitaria. Evaluación de un proyecto de investigacción-acción sobre el desamparo social de los menores. Reifop, 16 (3), 11-32.

Maslow, A. H. (1943). A Theory of Human Motivation. Psychological Review, 50, 370-396.

Peiró i Gregori, S. (2000). Educar en función de los valores. Alicante: Publicaciones de la Universidad de Alicante.

Peiró i Gregori, S. (2005). Indisciplina y violencia escolar. Alicante: Instituto Alicantino de Cultura «Juan Gil-Albert».

Peiró i Gregori, S. (ed.) (2013). Los valores en la educación. Madrid: Dykinson.

Penalva, A., Hernández, M.A. \& Guerrero, C. (2013). La gestión eficaz del docente en el aula. Reifop, 16 (2), 7791. Enlace web: http://www.aufop.com. [30-01-2014]

Penas, S. (2008). Aproximación a los valores y estilos e vida de los jóvenes de 13 y 14 años de la provincia de A Coruña. Universidad de Santiago de Compostela: Servicio de Publicaciones.

Prellezo, J.M., Nanni, C. Malizia, G. (1997). Dizionario di Scienze dell'educazione. Torino: Elle $\mathrm{Di}$ Ci.

Proyecto ICCS 2009 (International Civic and Citizenship Education Study). Enlace web: http:// www.iea.nl. [12-06-2013]. 
Recomendaciòn del Consejo de 18.12.2006. Enlace web: http://www.eur-lex.europea.eu. [1206-2013].

Rossini, V. (2011a). Climi di classe e stili educativi. In C. Gemma \& R. Pagano (a cura di). In principio...la ricerca. Temi e voci di un'esperienza di formazione (pp. 35-44). Milano: Franco Angeli.

Rossini, V. (2011b). Convivenza scolastica e formazione docente. In Elia G. (a cura di). Scuola e Mezzogiorno. Il Sud si interroga e propone (pp.282-296). Bari: Progedit.

Rueff, J. (1964). El orden social. Madrid: Aguilar.

Spaemann, R. (2001). Grenzen. Zur ethischen Dimension des Handelns. Stuttgart: Klett-Cota.

Thomas, W. H. \& Znaniecki, F. (1920). The Polish Peasant in Europe and America: Monograph of an Immigrant Group . Chicago: University of Chicago Press.

Weber, M. (2004). Economía y sociedad. Esbozo de sociología comprensiva. México: FCE.

Wojtyla, K. (2011). Persona y acción. Madrid: Palabra.

\section{Autores}

Valeria Rossini, Universidad de Bari (Italia).

Investigadora en Educación. Ahora es profesora de Pedagogía General y de Educación Especial en el curso de la licenciatura en Ciencias y técnicas psicológicas. Pertenece al Colegio de Profesores del Doctorado de "Dinámica formativa y educación a la política". Ha publicado dos libros y numerosos ensayos y artículos en libros y revistas nacionales e internacionales acerca de la educación infantil, la formación docente, la educación de los padres, la inclusión escolar.

Salvador Peiró-i-Gregòri, Universidad de Alicante (España).

Desde $1997 / 98$ ejerce en la Universidad de Alicante, en donde dirige un equipo de investigación sobre indisciplina, valores y violencia en educación. Ha publicado unos 70 libros y capítulos de libro, más de 80 artículos en revistas científicas, más de 200 artículos en medios de prensa diaria, y sobrepasa los ciento cincuenta eventos nacionales e internacionales. Ha fundado algunas asociaciones científicas, ha sido Director del Departamento de Pedagogía, y de la Revista de Educación de la Universidad de Granada. 